\title{
A Microfluidic Lab-on-a-Disc (LOD) for Antioxidant Activities of Plant Extracts
}

\author{
Nurhaslina Abd Rahman 1,2, Fatimah Ibrahim 1,2,*, Mohammad M. Aeinehvand 2,3, \\ Rohana Yusof ${ }^{4}$ and Marc Madou ${ }^{2,5}$ \\ 1 Department of Biomedical Engineering, Faculty of Engineering, University of Malaya, \\ 50603 Kuala Lumpur, Malaysia; haslin42@siswa.um.edu.my \\ 2 Centre for Innovation in Medical Engineering, Faculty of Engineering, University of Malaya, \\ 50603 Kuala Lumpur, Malaysia; m.aeinehvand@itesm.mx (M.M.A.); mmadou@uci.edu (M.M.) \\ 3 School of Engineering and Sciences, Tecnologico de Monterrey, Av. Eugenio Garza Sada 2501 Sur, \\ 64849 Monterrey, NL, Mexico \\ 4 Department of Molecular Medicine, Faculty of Medicine, University of Malaya, \\ 50603 Kuala Lumpur, Malaysia; rohana@um.edu.my \\ 5 Department of Biomedical Engineering, University of California, Irvine, CA 92697, USA \\ * Correspondence: fatimah@um.edu.my; Tel.: +60-379-676-818
}

Received: 13 January 2018; Accepted: 19 March 2018; Published: 21 March 2018

\begin{abstract}
Antioxidants are an important substance that can fight the deterioration of free radicals and can easily oxidize when exposed to light. There are many methods to measure the antioxidant activity in a biological sample, for example 2,2-diphenyl-1-picrylhydrazyl (DPPH) antioxidant activity test, which is one of the simplest methods used. Despite its simplicity, the organic solvent that has been used to dilute DPPH is easily evaporated and degraded with respect to light exposure and time. Thus, it needs to be used at the earliest convenient time prior to the experiment. To overcome this issue, a rapid and close system for antioxidant activity is required. In this paper, we introduced the Lab-on-a-Disc (LoD) method that integrates the DPPH antioxidant activity test on a microfluidic compact disc (CD). We used ascorbic acid, quercetin, Areca catechu, Polygonum minus, and Syzygium polyanthum plant extracts to compare the results of our proposed LoD method with the conventional method. Contrasted to the arduous laborious conventional method, our proposed method offer rapid analysis and simple determination of antioxidant. This proposed LoD method for antioxidant activity in plants would be a platform for the further development of antioxidant assay.
\end{abstract}

Keywords: Lab-on-a-Disc (LoD); centrifugal microfluidic CD; plant antioxidant activity; antioxidants; DPPH

\section{Introduction}

Free radicals are atoms with unpaired valence electrons that cause its chemical instability and reactivity. The valence electron of one atom accepts an electron from another molecule, which forms a new free radical. This process repeats itself, creating a free radical cascade. When free radical production becomes excessive in the body, it may cause oxidative stress, a condition whereby the body cannot counter-attack the production of free radicals, leading to cellular damage and cell death [1]. Free radicals have been implicated in many diseases, such as diabetes, hypertension, Parkinson's, Alzheimer's, and heart diseases [2-6]. Additionally, free radical production increases as the individual gets older, thereby accelerating the aging process [7].

Antioxidants are molecules that can donate electrons to stabilize free radical species. They play many important roles in daily life. For example, antioxidants are hypothesized to prevent the deleterious effects of free radicals on cells [8]. In the medical field, antioxidants have been used as 
an alternative disease therapy for diabetes mellitus, reperfusion injury, and inflammatory diseases. They also help to prevent atherosclerosis and carcinogenesis in the human body [9]. In the food industry, antioxidants can prevent the deterioration of food constituents and prevent it from spoiling. In addition, antioxidants absorb ultraviolet (UV) radiation, which minimizes the risk of skin cancer [10]. There are many potential natural chemical constituents that can be exploited from plants, such as medical plants containing high antioxidant properties, which are scientifically significant in the human body [11].

There are many methods to determine the antioxidant properties in a sample. Each of the methods work differently based on the chemical mechanism, among which, the 2,2-diphenyl-1-picrylhydrazyl (DPPH) antioxidant activity test is the most common and easiest method used to determine the antioxidant activity in a sample [12]. The DPPH antioxidant assay has been used widely to determine various antioxidant activities in food, beverages, and plants. Furthermore, it is also used to evaluate the antioxidant activity of nutrients in the human body [8]. DPPH is a stable, deep purple free radical powder, which will change to a pale yellow after reacting with the antioxidant. The free radical DPPH will react with the antioxidant, which acts as a hydrogen donor that stabilizes DPPH.

Despite the simplicity of the DPPH antioxidant assay, DPPH itself is easily decomposed over time and is highly sensitive to changes in temperature, $\mathrm{pH}$, and light exposure $[13,14]$. The DPPH solutions need to be diluted in organic solvent and prepared fresh before running the antioxidant activity test. According to Deng et al. [15], DPPH solution needs to be used within two hours of preparation to prevent the decomposition of the DPPH solution. It is also reported that the DPPH absorbance decreases within 90 min with changes in temperature. The absorbance of DPPH solution will also decrease when exposed to light within two hours, while in the dark, there are no significant changes in DPPH absorbance.

Many fundamental bio-assay methods are carried out manually. The experimental steps are often time-consuming and require expert skills. The amalgamation of human-prone error, especially in the pre-analytical phase, may also cause incorrect interpretations or false positive results in the analytical and post-analytical phases [16]. One of the most common pre-analytical errors is caused by pipetting error. The pipetting steps are the most common repetitive tasks for laboratorians; however, this step is often taken for granted. The pipetting steps, especially the microscale volumes, are very crucial and accuracy must be ensured to prevent erroneous results. Additionally, organic solvents evaporate easily and may contribute to volume loss. Errors also happen due to the repetitive steps and duplications when laboratorians are handling large amounts of samples.

Lab-on-a-Disc (LoD), which are also known as centrifugal microfluidic, are a part of the microfluidic device in the form of a disc with a spinning motor. LoD is a part of the Micro Total Analysis System ( $\mu$ TAS), which offers miniaturization and automation of most of the chemical and biological analysis systems. LoD offers many advantages, such as simplicity of the assay procedure, fast results, and cost efficiency. LoD has automated many bioassays, such as Enzyme-linked Immunosorbent Assay (ELISA) and the loop-mediated isothermal amplification (LAMP) assay [17,18]. When compared to lab-on-a-chips (LoCs) that require syringe pumps, LoD runs in a closed system and has an "all-in-one disc" assay procedure. LoD decreases the amount of reagent, sample usage and total processing time during the experiments. Subsequently, it also offers parallel or sequential loading of solutions. LoD skips multiple sample preparations and pipetting steps, which reduces human-prone handling error.

Jungwoo et al. [8] have demonstrated an LoC platform to evaluate the liver metabolism of antioxidants in food. He is focusing on mimicking the human liver metabolism and the detection of subsequent metabolized antioxidant food components. The design consist of a two microfluidic compartment; the first part contain human liver enzyme that mimic liver metabolism and the second part is DPPH detection of antioxidant activity of food components. In his design, the liver enzyme fractions were immobilized and the reaction with the DPPH solution were tested. Meanwhile, Xuhua et al. have used hydrogen peroxide techniques on a chip and to screen the antioxidant capacity 
by using herbal extracts [19]. His focused is more on the fabrication of the chip by using the thin-film organic photodiodes and the chemiluminescence detection of the antioxidant in the herbal extracts.

In this paper, we present an approach of LoD method for antioxidant plant activities. We offer automation and sample miniaturization of the DPPH antioxidant activity test with parallel sequential sample loading and mixing. Our proposed LoD method has been tested on ascorbic acid, quercetin, Areca catechu, Polygonus minus, and Syzygium polyanthum plant extract.

\section{Materials and Methods}

\subsection{Plant Materials Preparation}

The plants A. catechu, P. minus and S. polyanthum were collected from Selangor and Perak, Malaysia. The plants were chosen based on their reported high antioxidant activity among local plant species [20-22]. The plants were dried in a laboratory oven at $40{ }^{\circ} \mathrm{C}$ until crisp, then pulverized into a powder using a mechanical grinder. The ground powder was then soaked and shaken in 95\% ethanol for $48 \mathrm{~h}$. The solvent mixture was then filtered using Whatman No. 1 filter paper to eliminate the plant debris. After this, the excess solvents were eliminated using a rotary evaporator (Buchi Rotavapor R-114, Büchi Labortechnik AG, Flawil, Swizerland), then freeze-dried, and stored for $20{ }^{\circ} \mathrm{C}$ until further use in the experiment.

\subsection{DPPH Conventional Antioxidant Activity Test}

The antioxidant activity of the samples were determined using the method described by Saha et al. [23], with some modification. The deep purple colorimetry reduction of DPPH was determined by using a spectrophotometer (Epoch, BioTek Instruments, Winooski, VT, USA) at $517 \mathrm{~nm}$ with three repeated measurements. First, the plant extracts were diluted to four final concentrations of $25,50,75$, and $100 \mathrm{mg} / \mathrm{mL}$, to make $1 \mathrm{~mL}$ sample solutions in ethanol. Then, $1 \mathrm{mM}$ of DPPH in methanol was prepared and added to the test solution and left to incubate for $30 \mathrm{~min}$ at room temperature. For the control experiment, ethanol was added with DPPH. Finally, the absorbance values were measured and the antioxidant activities were calculated using the following equation.

$$
\text { Antioxidant activity }=\frac{\text { Control }- \text { Test Sample }}{\text { Control }}
$$

\subsection{Microfluidic Compact Disc (CD) Design and Fabrication}

The photoprotective microfluidic was designed using computer-aided design software (AutoCAD), as shown in Figure 1a. The photoprotective microfluidic CD consists of five layers: three black Polymethyl Methacrylate (PMMA) and two pressure sensitive adhesive (PSA) layers. The first layer is a transparent PMMA disc and contains injection/venting holes. A black adhesive film is used to cover the PMMA disc except the reaction chambers. The second layer is PSA and the third layer is a black PMMA disc with the microfluidic channel and chamber features engraved on it. The fourth layer is PSA and the fifth layer of the microfluidic CD is made of black PMMA. The CD features details can be seen in the Supplementary section.

All of the engraving of microfluidic channel features was done using a Computer Numerical Control (CNC) machine VISION 2525 by Vision Engraving and Routing Systems, USA, as shown in Figure 1b. The engraved microfluidic channel features three chambers: a DPPH chamber, plant extract chamber, and reaction chamber. The DPPH chamber was connected to the valve to control the flow of DPPH solution to the reaction chamber. The inlet holes and microfluidic features in the PSA layers were cut using a cutter plotter machine (GCC P2-60/PUMA II, by GCC, Taiwan). Each layer of microfluidic $C D$ is press-bonded together using a custom-made pressing tool.

Figure 1c shows the whole experimental set up, which consists of computer controlling systems and custom LoD spinning test systems. The centrifugal motor and the high-speed camera were connected to the computer to control the microfluidic CD rotating speed (rpm) and visualization, 
respectively. The reflector attached to the microfluidic $C D$ will provide the signal to the digital RPM meter to determine the $C D^{\prime}$ s rotation speed.
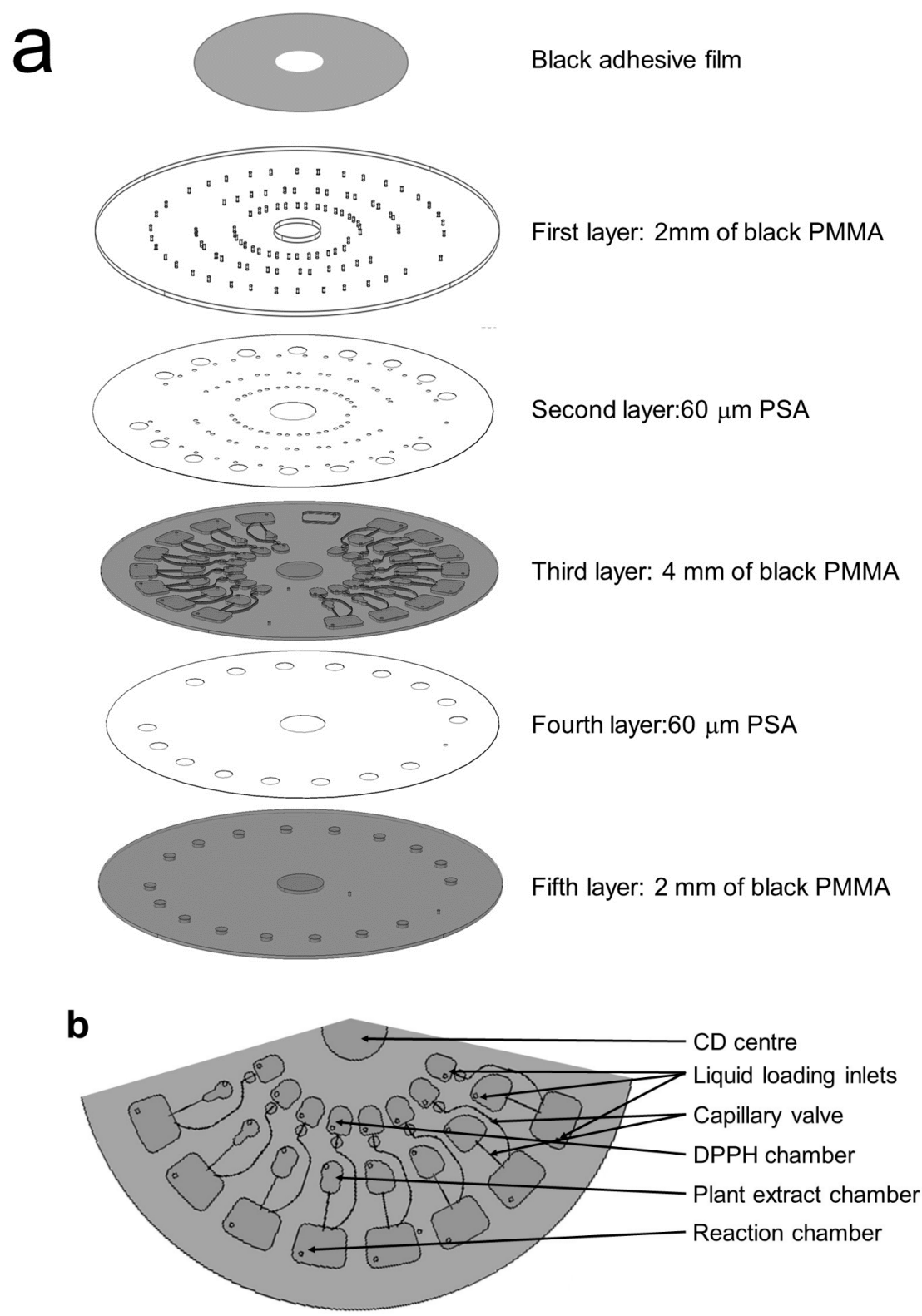

Figure 1. Cont. 


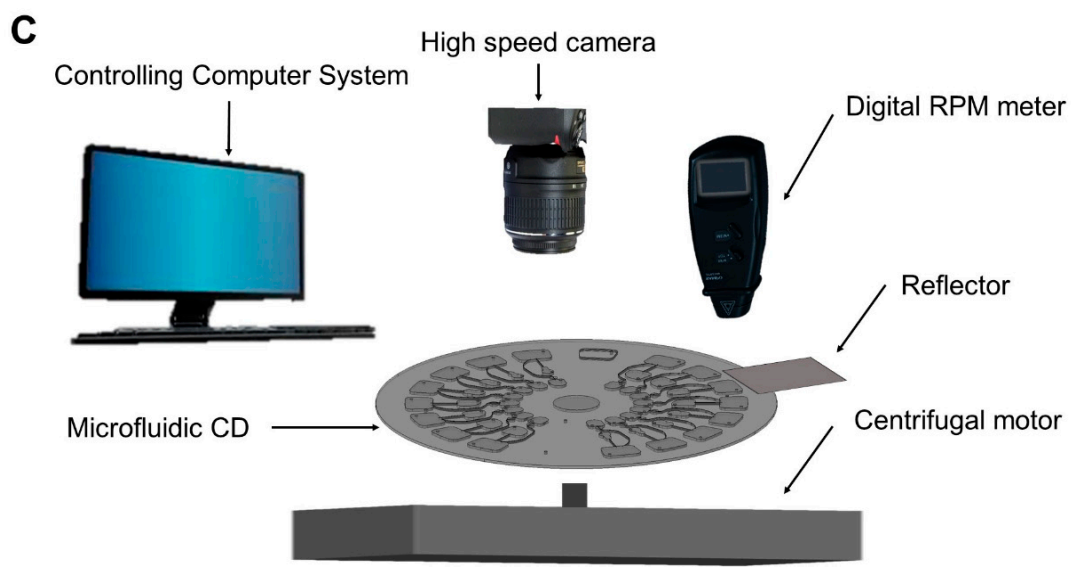

Figure 1. (a) An exploded view of the photo-protective layers of the microfluidic compact disc (CD). The CD is consist of five layers. The first, third, and the fifth layer was made from black PMMA layer. While the second and the third layer was made from PSA; (b) A top view of the third layer of the antioxidant microfluidic CD with different specification of chamber. The plant extract chamber was varied in four different sizes and duplicated for two consecutive concentrations; (c) The whole experimental set up. The whole experiment set up consists of the computer controlling systems and the custom Lab-on-a-Disc (LOD) spinning test system. The centrifugal motor and the high-speed camera were connected to the computer to control the CD speed (rpm) and visualisation. The reflector will give the signal to the digital RPM.

\subsection{Integrated Microfluidic DPPH CD Operations}

After preparing the plant extract (as discussed in the previous section), the plant extracts along with the two-standard reagent of antioxidant (ascorbic acid and quercetin) were then preloaded into the plant extract chambers. The plant extract chambers in the middle were designed in four different sizes in duplicate for 25, 50, 75, and $100 \mathrm{mg} / \mathrm{mL}$ plant extract. Meanwhile, as shown in Figure 1b, the reaction chamber was located at the bottom of the microfluidic $\mathrm{CD}$ and prefilled with the solvents. It is the final chamber for the mixture of the solvent, plant extract, and the DPPH. The DPPH chamber connected with a valve was designed to occupy $1 \mathrm{mM} \mathrm{DPPH}$. After all of the reagents are preloaded to the designated chamber, the holes were sealed with PCR sealing tapes. The centrifugal microfluidic process starts after all the liquids have been loaded in to the designated chamber. The spinning operation is summarized in Table 1 . The spinning is stopped after $30 \mathrm{~min}$ and the absorbance reading were measured.

Table 1. Spinning program of the microfluidic CD.

\begin{tabular}{|c|c|c|c|c|}
\hline Step & Spinning Speed (rpm) & Time * & $\begin{array}{l}\text { Spinning } \\
\text { Direction }\end{array}$ & Process \\
\hline 1 & 0 & $1 \mathrm{~min}$ & nil & Sample preloading \\
\hline 2 & 300 & $30 \mathrm{~s}$ & Clockwise & $\begin{array}{l}\text { Plant extracts flowing to the reaction } \\
\text { chamber and emptied }\end{array}$ \\
\hline 3 & 800 & $15 \mathrm{~s}$ & Anticlockwise & $\begin{array}{l}\text { 2,2-diphenyl-1-picrylhydrazyl (DPPH) } \\
\text { solution flow out from the DPPH chamber } \\
\text { to the capillary valve and reached reaction } \\
\text { chamber }\end{array}$ \\
\hline 4 & 1400 & $\begin{array}{l}5 \mathrm{~min} \\
\text { interval to } \\
30 \mathrm{~min}\end{array}$ & $\begin{array}{l}\text { Clockwise and } \\
\text { anticlockwise }\end{array}$ & The disc is rotated for mixing purposes \\
\hline
\end{tabular}

* The time includes the acceleration time to reach the target speed and to change the rotation $(2 \mathrm{~s})$. 


\subsection{Absorbance Reading}

There are two devices used to read the antioxidant activity absorbance, which are a microplate reader and the $C D$ reader. The microplate reader was used to compare the antioxidant absorbance activity for both the conventional and $\mathrm{LoD}$ method reading over $5 \mathrm{~min}$ intervals up to $30 \mathrm{~min}$. The final solutions obtained from the conventional and $\mathrm{LoD}$ reaction methods were then transferred to the 96 well plates for the absorbance measurements.

Whereas, the $\mathrm{CD}$ reader (Figure 2) was used to measure the absorbance of the final biochemical solutions in the microfluidic CD 24, 25. The CD reader consisted of a $517 \mathrm{~nm}$ LED and photodiode to measure the light intensity passing through the solution. A microcontroller (Atmega328PU, Microchip Technology Inc., Chandler, AZ, USA) connected with the photodiode, calculates the absorbance of the light passing through the solutions. A stepper motor, a Hall Effect sensor and a magnet help to incorporate the alignment among the reaction chamber, LED, and photodiode. The CD reader structure was built using black PMMA to reduce the optical noise.

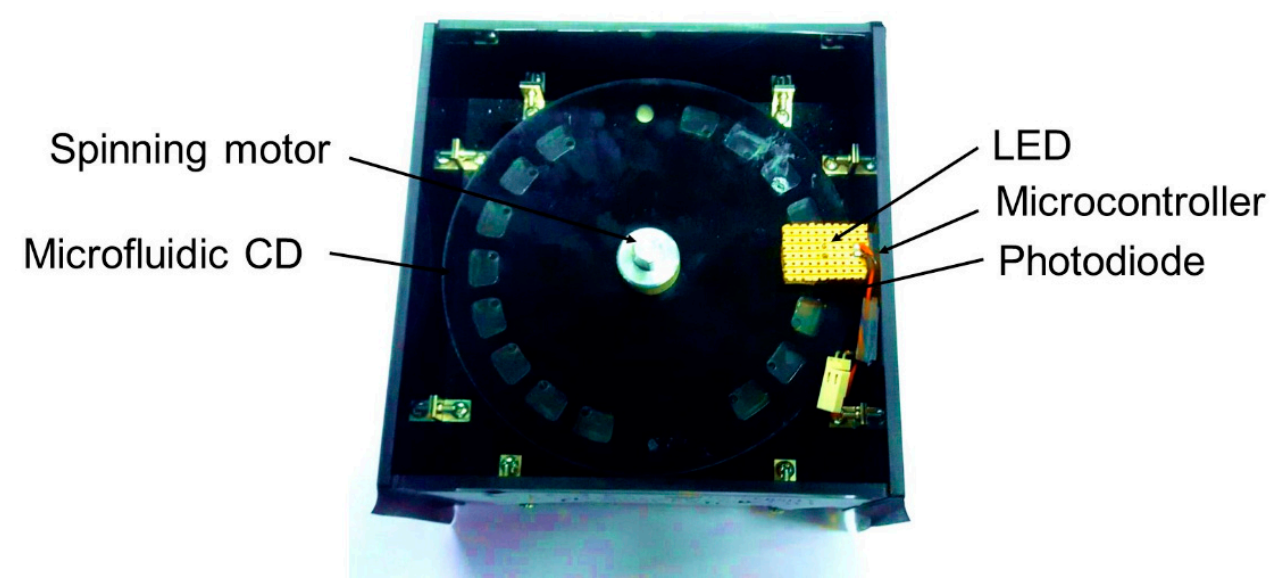

Figure 2. The $C D$ reader is used to read the final absorbance directly from the $C D$. The results were then compared to the microplate reader.

\section{Results and Discussion}

\subsection{Microfluidic CD Operations}

Figure 3 shows the actual images from the complete process of microfluidic CD operations, sequential loading, and mixing. For display purposes, the microfluidic $C D$ was fabricated using clear PMMA. The schematic illustrations on the left side are drawn to show and explain the experiments, with corresponding images captures from the video of the high speed camera. In the first step, Figure 3a has shown the liquids have been preloaded into the designated chambers i.e., DPPH solution in the DPPH chamber, plant extract solution in the plant extract chamber, and solvents in the reaction chamber. To start the experiment, the speed of the centrifugal motor was increased slowly. During this process, the plant extract solution started to flow into the capillary valve. This process is shown in Figure 3b. Subsequently, after the rotation speeds have reached $300 \mathrm{rpm}$, all the plant extract chambers have been emptied and the solution has been mixed in the reaction chamber with the solvent (as shown in Figure 3c). In Figure 3d, the speed was increased, which makes the DPPH solution flow out from its chamber to the capillary valve. At $800 \mathrm{rpm}$, it was noticed that all the DPPH solution was emptied from the chamber as the solution moved to the reaction chamber, which is shown in Figure $3 \mathrm{e}$. Finally, in Figure $3 \mathrm{f}$ the centrifugal speed was increased to $1400 \mathrm{rpm}$ to ensure all the solutions were mixed properly. 

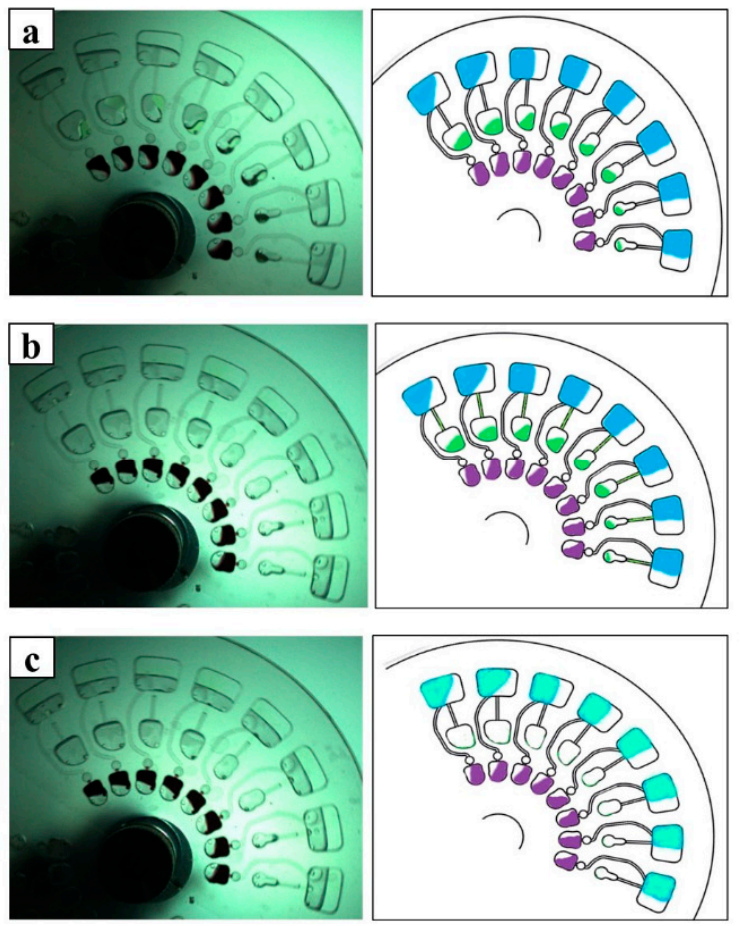
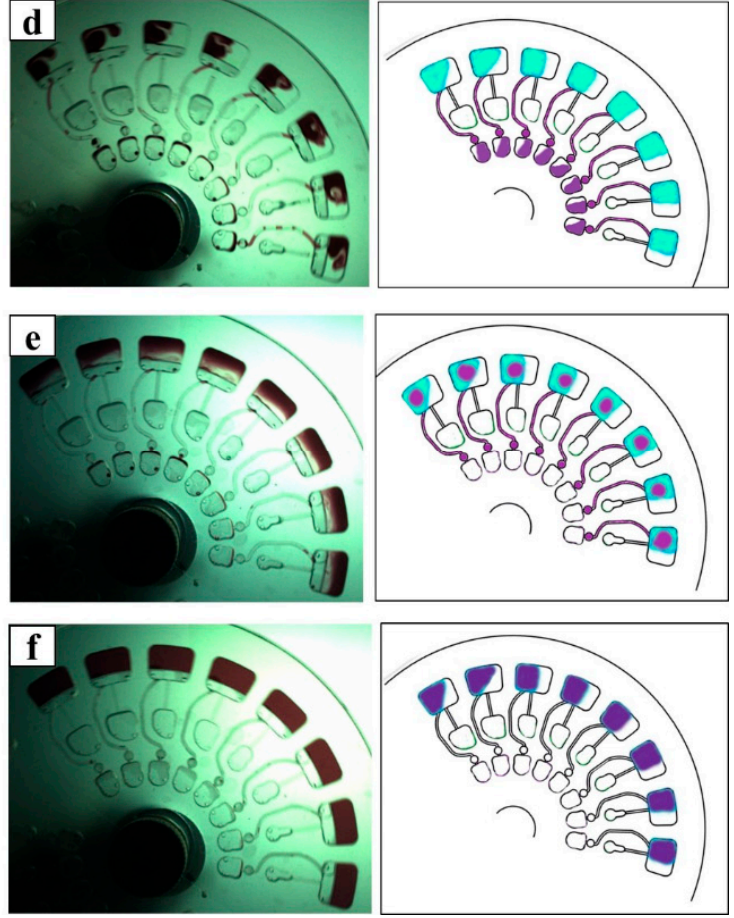

Figure 3. Entire sequences of the images and schematic illustrations in the microfluidics $C D$. (a) The initial of the experiment, speed 0 . (b) The plant extract solution started to flow into the capillary valve; speed has been increased from 0 to $300 \mathrm{rpm}$. (c) The plant extract chambers have been emptied; speed $300 \mathrm{rpm}$. (d) DPPH solution flow out from its chamber to the capillary valve; speed has been increased slowly from 300 to $800 \mathrm{rpm}$. (e) The DPPH chamber was emptied; speed $800 \mathrm{rpm}$. (f) All of the solutions were mixed properly in the reaction chamber; speed $1400 \mathrm{rpm}$.

Our proposed method has minimized human operations in the DPPH antioxidant test, the repetitive pipetting, loading, and mixing steps can be skipped by flow control mechanism of the microfluidic CD. Basically, in our microfluidic CD design, the capillary passive valves were used to control the sequential fluidic flow and the mixing of the liquids. The manipulation of the chamber position, centrifugal force and the capillary valve have determined the sequences of fluids flows to the reaction chamber, the details and analysis of the valves burst frequency is described by Thio et al. [24] and Kazemzadeh et al. [25].

Thio et al. and Kazemzadeh et al. in their paper have been discussed extensively about the theory of the liquid flows inside the passive capillary valves toward the target chamber (in this paper is reaction chamber). In order of the liquid to move from one chamber to another, the capillary pressure need to be overcome by increasing the speed of the motor (centrifugal pressure will have increased parallelly). In this paper, the location and the geometry of the plant extract chamber and the DPPH chamber on the microfluidic $C D$ have enable the liquid flows manipulation into the reaction chamber.

\subsection{Comparisons of Conventional and LoD DPPH Antioxidant Activity Method}

In this section, the comparison results of the conventional and LoD DPPH antioxidant methods are shown and discussed. All of the samples were tested for antioxidant activity by using LoD method and compared with the conventional DPPH antioxidant activity test for $30 \mathrm{~min}$ as this is the standard time and common procedures practises in the conventional DPPH assay [26]. Subsequently, the results were further analyzed in $5 \mathrm{~min}$ intervals up to $30 \mathrm{~min}$. For the purposes of analysis, a repeated ANOVA measure by IBM SPSS statistical software version 24 was used.

In Figure 4, the comparisons of the ascorbic acid, quercetin, A. catechu, P. minus, and S. polyanthum activity antioxidant has been presented between conventional and LoD method at each concentration. 
The results showed that there was a significant difference of $* \leq 0.05$ between the conventional and LoD method at each concentration. What can be clearly observed in Figure 4 is the levels of the DPPH activity in the LoD method are consistently higher than the conventional method. In the conventional method, the reaction is let to stand for $30 \mathrm{~min}$ without any force being applied on it. Whereas, in the LoD method, mixing scheme has been applied which involves the combination of centrifugal force, Euler force, and Coriolis force. Centrifugal and Euler force is important for the automated liquid handling processing. While Coriolis force creates the stirring effect in the reaction chamber that leads to better liquid homogenization and diffusion of the particles.

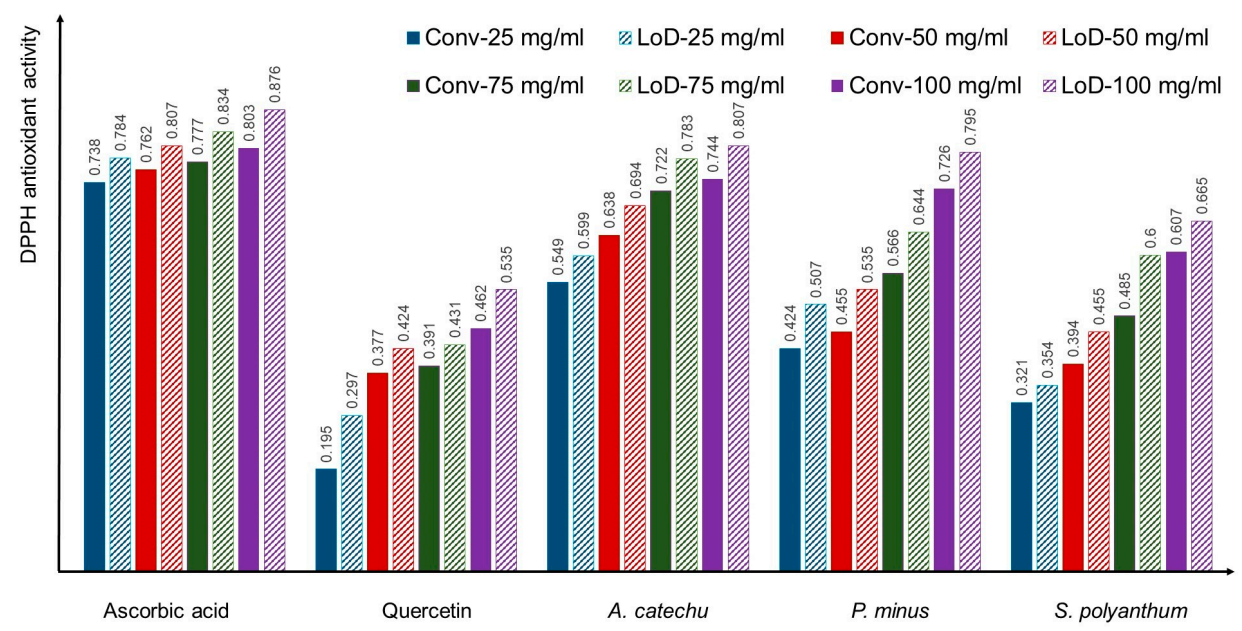

Figure 4. Comparison of antioxidant activity in conventional and LoD method at different concentrations.

Efficacious sample mixing accelerate the chemical reaction and decrease the time of assay [27]. In a chemical reaction, the ability to create a fast homogenous reactant mixture is crucial, especially in the small sample volume. For rapid chemical reactions to occur, a fast kinetics reaction and high contact frequency between particles can be increased with a good mixing efficiency, which this phenomena has been provided in the LoD platform [28,29]. Our proposed method has introduced a passive mixers which maximize the area of the chemical reaction to occur in the reaction chamber. With the constant application of Coriolis stirring effect by the CD rotation, the chemical reaction between the plant extract and the DPPH has been enhanced, which in contrast does not happen in the conventional method.

\subsubsection{Ascorbic Acid}

In Figure 5, it can be seen that, the DPPH activity value with the LoD method at 10 min gave the same activity level as the conventional method for $25 \mathrm{mg} / \mathrm{mL}, 50 \mathrm{mg} / \mathrm{mL}$, and $75 \mathrm{mg} / \mathrm{mL}$ ascorbic acid at $30 \mathrm{~min}$. On the other hand, $100 \mathrm{mg} / \mathrm{mL}$ with the LoD method of ascorbic acid took only $5 \mathrm{~min}$ to reach the antioxidant activity level of conventional method equal to $30 \mathrm{~min}$. On average, the LoD method that was tested with ascorbic acid was able to produce similar measurements when compared to the conventional method with an analysis reduction time of $21.25 \mathrm{~min}$ i.e., the LoD method duration was $5 \mathrm{~min}$ at $100 \mathrm{mg} / \mathrm{mL}$, whereas the conventional method took $30 \mathrm{~min}$. The mean, SE, and SD for the different concentrations and different time intervals were clearly seen in Appendix A. The small values of the SE and SD indicate that the results are repeatable and precision of the mean value [30]. 


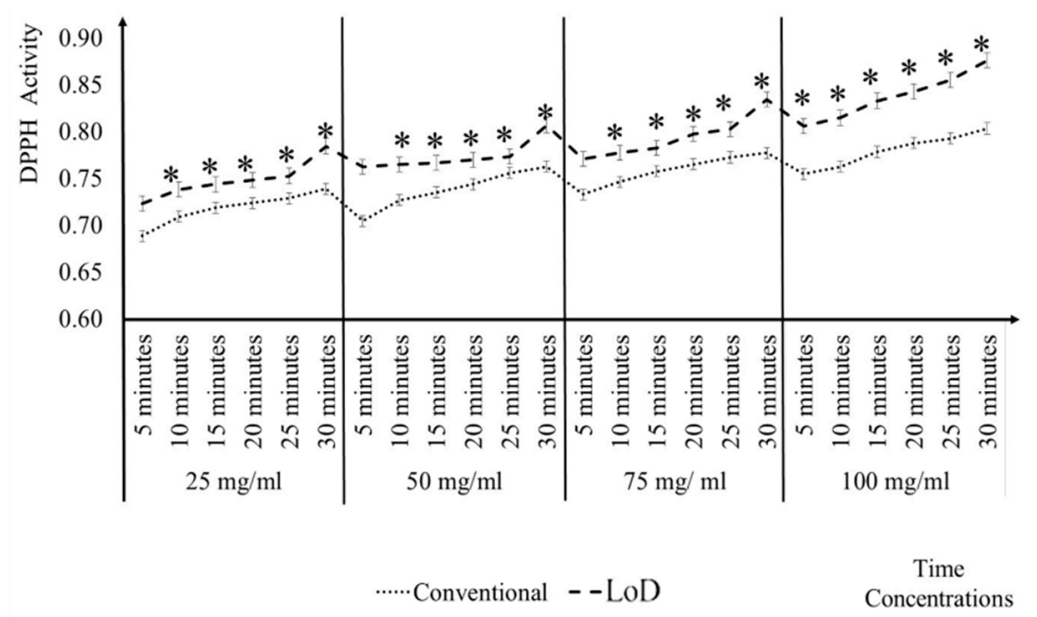

Figure 5. Comparison of ascorbic acid antioxidant activity in the conventional and LoD method by 5 min interval.

\subsubsection{Quercetin}

Figure 6 shows that, its takes $20 \mathrm{~min}$ for $25 \mathrm{mg} / \mathrm{mL}$ and $15 \mathrm{~min}$ for $50 \mathrm{mg} / \mathrm{mL}$ and $75 \mathrm{mg} / \mathrm{mL}$ of Quercetin to give the comparable antioxidant activity level of the DPPH conventional methods at $30 \mathrm{~min}$. However, for $100 \mathrm{mg} / \mathrm{mL}$ Quercetin, it only took $10 \mathrm{~min}$ to give the same antioxidant activity level as the DPPH conventional methods at $30 \mathrm{~min}$. On average, the LoD method accelerated the analysis time for Quercetin by $15 \mathrm{~min}$ when compared to the conventional methods at $30 \mathrm{~min}$. The mean, SE, and SD for the different concentrations and different time intervals can be clearly seen in Appendix B.

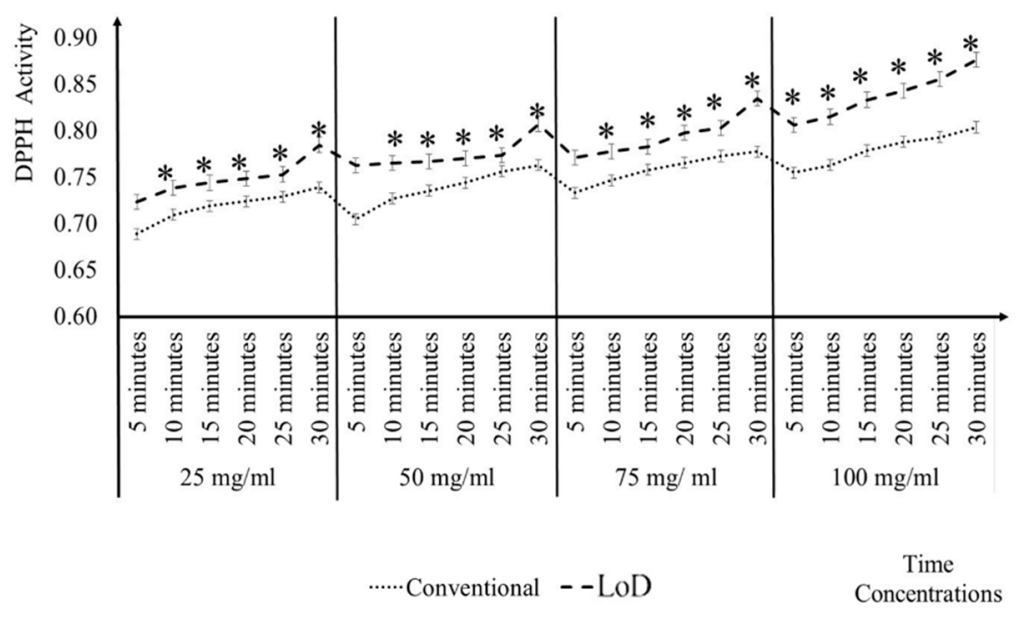

Figure 6. Comparison of quercetin antioxidant activity determined using conventional and LoD methods by 5 min time intervals.

\subsubsection{A. catechu}

Figure 7 shows that it took $20 \mathrm{~min}$ and $15 \mathrm{~min}$ for $25 \mathrm{mg} / \mathrm{mL}$ and $50 \mathrm{mg} / \mathrm{mL}$, respectively, to give the same antioxidant activity level as the DPPH conventional methods at $30 \mathrm{~min}$. Meanwhile, for $75 \mathrm{mg} / \mathrm{mL}$ and $100 \mathrm{mg} / \mathrm{mL}$, at $10 \mathrm{~min}$ the LoD method was sufficient to give the same antioxidant activity as the DPPH conventional methods at $30 \mathrm{~min}$. On average, the LoD method that was tested with $A$. catechu plant extract gave a result that was $16.25 \mathrm{~min}$ faster when compared to the conventional methods at $30 \mathrm{~min}$. The analysis can be seen in Appendix C. 


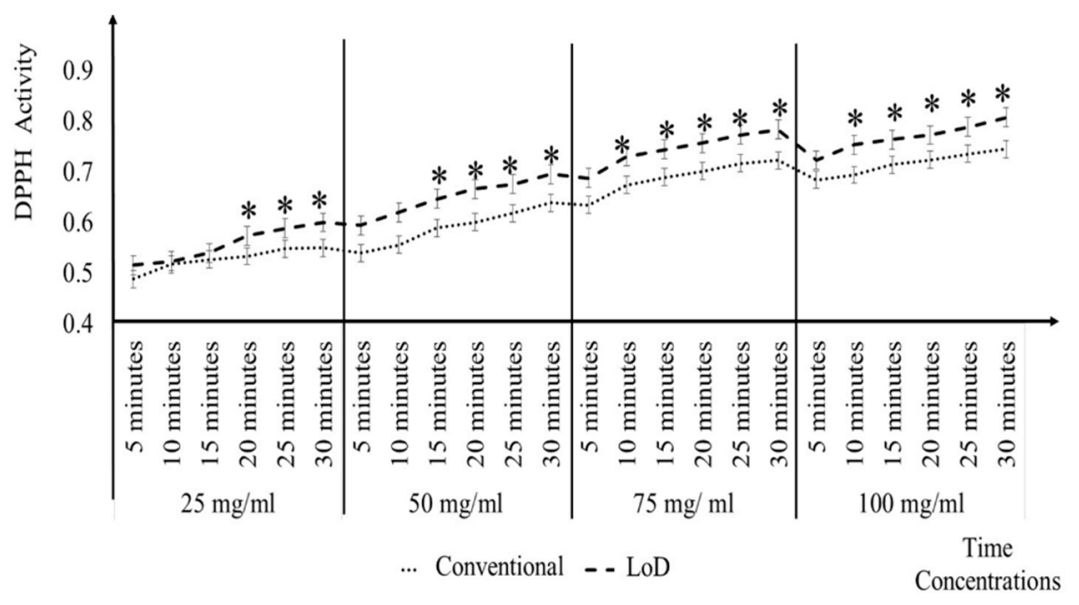

Figure 7. Comparison of $A$. catechu antioxidant activity determined using conventional and LoD methods by 5 min time interval.

\subsubsection{P. minus}

The results in Figure 8 shows that $5 \mathrm{~min}$ for $25 \mathrm{mg} / \mathrm{mL}, 50 \mathrm{mg} / \mathrm{mL}, 75 \mathrm{mg} / \mathrm{mL}$, and $100 \mathrm{mg} / \mathrm{mL}$ in the LOD method are sufficient to give the same antioxidant activity as the same concentrations at $30 \mathrm{~min}$ for the DPPH conventional methods. The analysis of the mean, SE and SD for different concentrations and different time intervals can be clearly seen in Appendix D.

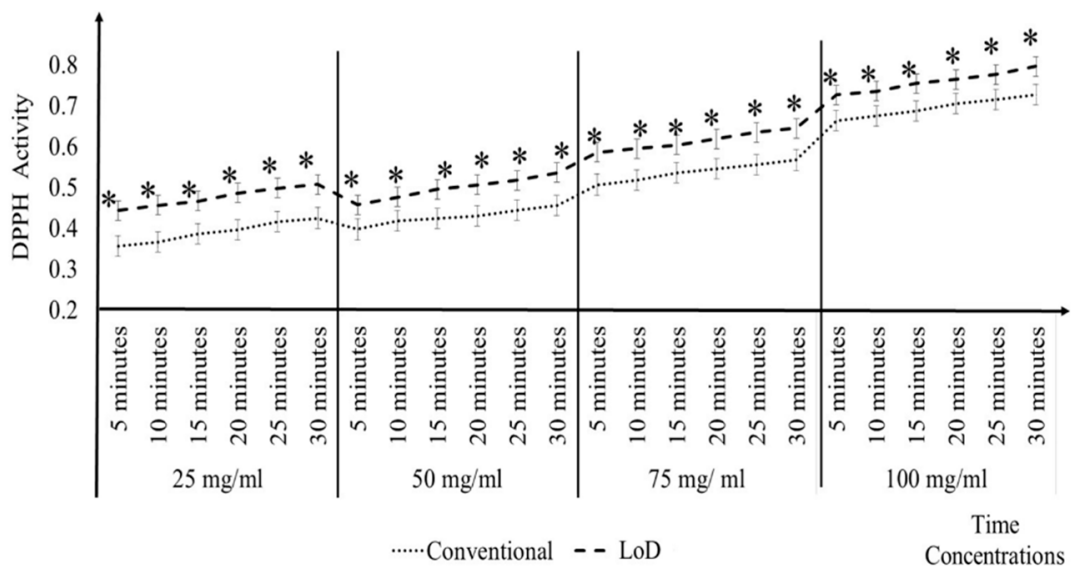

Figure 8. Comparison of P. minus antioxidant activity determined using conventional and LoD methods 5 min time interval in each concentration.

\subsubsection{S. polyanthum}

The results in Figure 9 indicate that the LoD method was able to give the same antioxidant level as early as $5 \mathrm{~min}$ at $50 \mathrm{mg} / \mathrm{mL}, 75 \mathrm{mg} / \mathrm{mL}$, and $100 \mathrm{mg} / \mathrm{mL}$. By using the LoD method, it is believed with the increase of the concentrations, the time to reach the same activity level as the conventional method will be decreased. On average, the LoD method that was tested with S. polyanthum plant extract gave a result that was $21.25 \mathrm{~min}$ faster when compared to the conventional methods. Refer Appendix E for the analysis. 


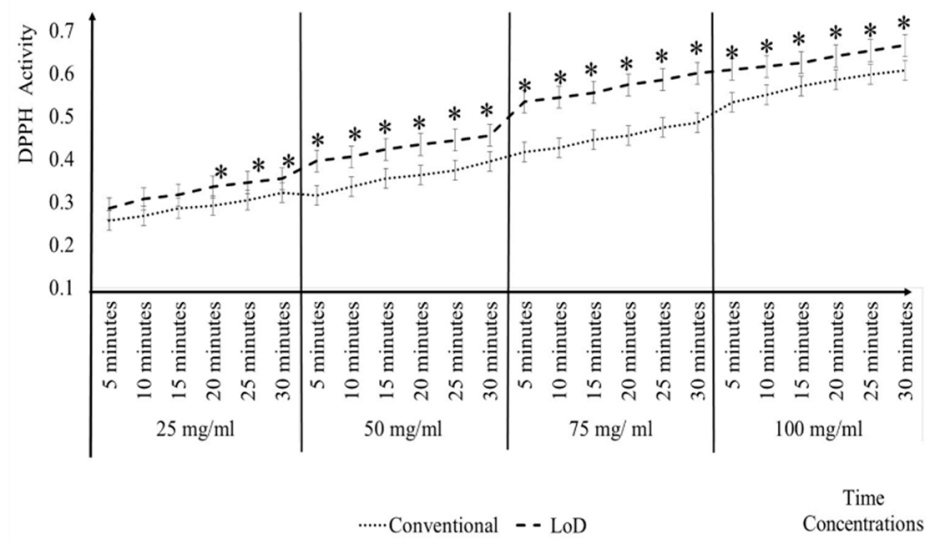

Figure 9. Comparison of S. polyanthum antioxidant activity in conventional and LoD method by 5 min time interval.

The results has been shown in Figures 4-8, where the reading does not reach plateau state in the microfluidics CD method, in contrast to the finding with Phonchai et al., which have shown a plateau DPPH activity reading with respective mixing time [31]. However, in contrast many researchers have also agreed that the kinetics reaction in DPPH assay may persist from minutes to hours. Although it is recommendable to allow for the reaction and measurement until the plateau reading state, the diverse completion kinetic reaction time in DPPH have led us to use $30 \mathrm{~min}$ as a single standard measuring time [26,32,33]. Additionally, Xie et al. [13] have illustrated that, regardless of the incubation time, the assay definitely reflects the stoichiometry DPPH reaction with the antioxidant in the sample.

Referring to the Figures 5-9, it can be clearly seen that the time taken to measure the DPPH activity varies among different samples. For example, the analysis of $25 \mathrm{mg} / \mathrm{mL}$ solutions of ascorbic acid and quercetin using LoD method was able to give the comparable DPPH antioxidant activity to the conventional method at 10 and 20 min, respectively, meanwhile the LoD method reduced the time that was taken to analyse P. minus to $5 \mathrm{~min}$, when compared to the standard $30 \mathrm{~min}$ of the conventional method. This result may be explained by the fact that the purity, compound mixtures, different form, and weight of molecules that are present in a sample did play a role for diverse antioxidant activity. In this study, the plant extract have been used to test the LoD method, the presence of compound, such as phenol, may cause interference and affect the reading when compared to the actual activity, which expound the different activity time between each plant $[34,35]$.

The conventional DPPH method use a high concentrations of the plant extract (i.e., 100, 200, 400, $800 \mathrm{mg} / \mathrm{mL}$ ), which proportionally need large reagent volumes. The proposed method reduced the volume usage to the final of $200 \mu \mathrm{L}$ to provide a good range of detection as the conventional method [31]. Figure 10 shows a fitted correlation of the original conventional method and the proposed method reduced volume. The decreased amount of reagent and sample that were used in the experiments offer optimal advantages for the rare samples and limited reagent supply.

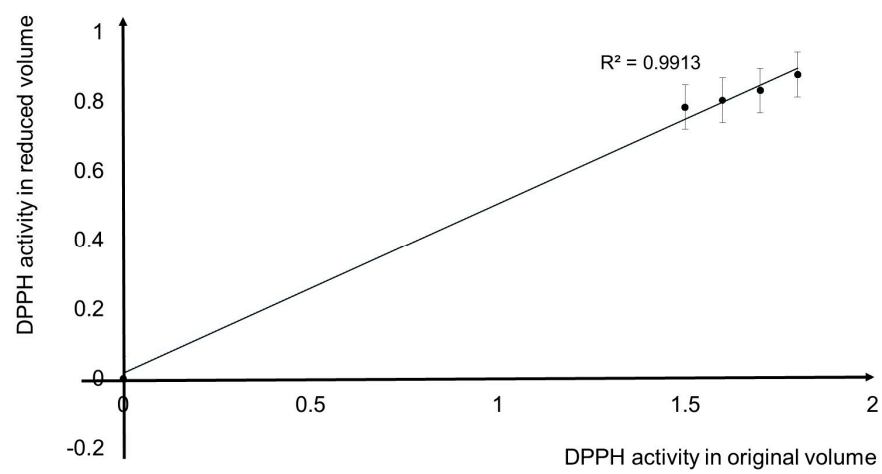

Figure 10. DPPH activity correlation between the original and reduced volume in proposed method. 


\subsection{Comparisons of CD Reader and the Conventional Microplate Reader}

The experimental work was carried out with a modified version of custom-made CD spin test system [36,37]. The readings were taken after a $30 \mathrm{~min}$ incubation for both the proposed LoD method and the conventional DPPH method. The data in Figure 11 shows a fitted coefficient and correlations $\left(\mathrm{r}^{2}=0.96\right)$ between the reading from the $\mathrm{CD}$ reader and the microplate reader, indicating a close relationship between the $C D$ reader and the microplate reader. Unlike the conventional method, the $\mathrm{CD}$ reader can read the absorbance of the entire sample directly from the microfluidic $\mathrm{CD}$. It is designed specially to work in complement with the microfluidic $\mathrm{CD}$, and the pipetting process of transferring the solutions to the 96 well plate has be eliminated. The process of transferring the solutions to the 96 well plate risks losing volume, which can be rectified by a closed and automated system, such as that used in the LoD platform. This CD reader endpoint reading system complete and support to the development of an integrated platform for the antioxidant microfluidics CD system.

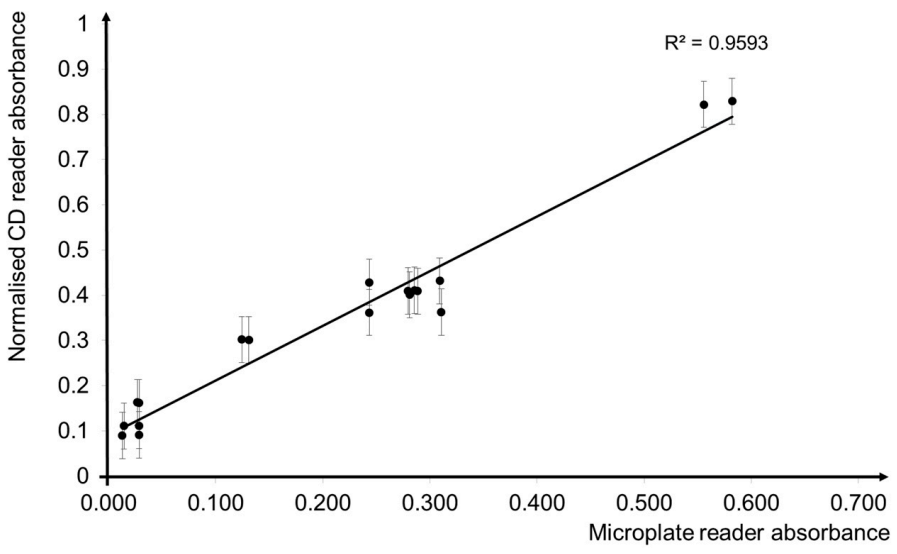

Figure 11. Graph of coefficient correlation between the CD plate reader and the microplate reader.

\section{Conclusions}

In this study, we have integrated the conventional DPPH antioxidant activity test by proposing a microfluidics CD method for the detection of antioxidant activities in plants. The sequential sample loading and mixing in a closed LoD system minimizes human error and volume loss due to manual pipetting procedures. The "load and run concept" in the proposed LoD method has omitted the repetitive pipetting, mixing, and loading steps in the DPPH conventional method. With four parallel concentrations and duplications that able been run simultaneously on the microfluidic $C D$, this study has shown that the proposed LoD method for antioxidant activities of the plant extracts procedures has been automated. Using this LoD technique not only it automates the processes but also helps to reduce the incubation time to five minutes, to reach the same antioxidant activity level as the conventional method of $30 \mathrm{~min}$. This approach would work as an application for antioxidant activity and will act as a platform for the determination of future antioxidant activity in a plants or another type of sample.

Supplementary Materials: The following are available online at http:/ / www.mdpi.com/2072-666X/9/4/140/s1.

Acknowledgments: This research was supported by University of Malaya Research Grant (UMRG: RP022B-14AFR) and Postgraduate Research Grant (PPP)-PG333-2016A.

Author Contributions: Nurhaslina Abd Rahman has conducted the experiments, conceptualized the idea of the paper, analyzed the results and written the manuscript. Mohammad M. Aeinehvand has contributed the LoD experiment and technical support. Fatimah Ibrahim secured the grant, proposed the project and was involved in the analysis of the results. Rohana Yusof was involved in the plant antioxidant activity experiments. Both Fatimah Ibrahim and Rohana Yusof supervised the work. Marc Madou provided the generalized the idea for the study.

Conflicts of Interest: The authors have no conflict of interest. 


\section{References}

1. Sinha, K.; Das, J.; Pal, P.B.; Sil, P.C. Oxidative stress: The mitochondria-dependent and mitochondria-independent pathways of apoptosis. Arch. Toxicol. 2013, 87, 1157-1180. [CrossRef] [PubMed]

2. Matsuda, M.; Shimomura, I. Increased oxidative stress in obesity: Implications for metabolic syndrome, diabetes, hypertension, dyslipidemia, atherosclerosis, and cancer. Obes. Res. Clin. Pract. 2013, 7, e330-e341. [CrossRef] [PubMed]

3. Dikalov, S.I.; Ungvari, Z. Role of mitochondrial oxidative stress in hypertension. Am. J. Physiol. Heart Circ. Physiol. 2013, 305, H1417-H1427. [CrossRef] [PubMed]

4. Dias, V.; Junn, E.; Mouradian, M.M. The role of oxidative stress in parkinson's disease. J. Parkinson Dis. 2013, 3, 461-491.

5. Yan, M.H.; Wang, X.; Zhu, X. Mitochondrial defects and oxidative stress in alzheimer disease and Parkinson disease. Free Radic. Biol. Med. 2013, 62, 90-101. [CrossRef] [PubMed]

6. Vanessa Fiorentino, T.; Prioletta, A.; Zuo, P.; Folli, F. Hyperglycemia-induced oxidative stress and its role in diabetes mellitus related cardiovascular diseases. Curr. Pharm. Des. 2013, 19, 5695-5703. [CrossRef]

7. Ashok, B.T.; Ali, R. The aging paradox: Free radical theory of aging. Exp. Gerontol. 1999, 34, $293-303$. [CrossRef]

8. Lee, J.; Choi, J.R.; Ha, S.K.; Choi, I.; Lee, S.H.; Kim, D.; Choi, N.; Sung, J.H. A microfluidic device for evaluating the dynamics of the metabolism-dependent antioxidant activity of nutrients. Lab Chip 2014, 14, 2948-2957. [CrossRef] [PubMed]

9. Maxwell, S.R.J. Prospects for the use of antioxidant therapies. Drugs 1995, 49, 345-361. [CrossRef] [PubMed]

10. Pisoschi, A.M.; Pop, A. The role of antioxidants in the chemistry of oxidative stress: A review. Eur. J. Med. Chem. 2015, 97, 55-74. [CrossRef] [PubMed]

11. Li, S.; Li, S.K.; Gan, R.Y.; Song, F.L.; Kuang, L.; Li, H.B. Antioxidant capacities and total phenolic contents of infusions from 223 medicinal plants. Ind. Crop. Prod. 2013, 51, 289-298. [CrossRef]

12. Gupta, V.K.; Sharma, S.K. Plants as natural antioxidants. Nat. Prod. Radiance 2006, 5, 326-334.

13. Xie, J.; Schaich, K.M. Re-evaluation of the 2,2-diphenyl-1-picrylhydrazyl free radical (DPPH) assay for antioxidant activity. J. Agric. Food Chem. 2014, 62, 4251-4260. [CrossRef] [PubMed]

14. Pyrzynska, K.; Pekal, A. Application of free radical diphenylpicrylhydrazyl (DPPH) to estimate the antioxidant capacity of food samples. Anal. Methods 2013, 5, 4288-4295. [CrossRef]

15. Deng, J.; Cheng, W.; Yang, G. A novel antioxidant activity index (aau) for natural products using the DPPH assay. Food Chem. 2011, 125, 1430-1435. [CrossRef]

16. Rana, S.V. No Preanalytical Errors in Laboratory Testing: A Beneficial Aspect for Patients; Springer: Berlin, Germany, 2012.

17. Hosseini, S.; Azari, P.; Aeinehvand, M.M.; Rothan, H.A.; Djordjevic, I.; Martinez-Chapa, S.O.; Madou, M.J. Intrant elisa: A novel approach to fabrication of electrospun fiber mat-assisted biosensor platforms and their integration within standard analytical well plates. Appl. Sci. 2016, 6, 336. [CrossRef]

18. Sayad, A.A.; Ibrahim, F.; Uddin, S.M.; Pei, K.X.; Mohktar, M.S.; Madou, M.; Thong, K.L. A microfluidic lab-on-a-disc integrated loop mediated isothermal amplification for foodborne pathogen detection. Sens. Actuators B Chem. 2016, 227, 600-609. [CrossRef]

19. Wang, X.; Amatatongchai, M.; Nacapricha, D.; Hofmann, O.; de Mello, J.C.; Bradley, D.D.; de Mello, A.J. Thin-film organic photodiodes for integrated on-chip chemiluminescence detection-application to antioxidant capacity screening. Sens. Actuators B Chem. 2009, 140, 643-648. [CrossRef]

20. George, A.; Ng, C.P.; O'Callaghan, M.; Jensen, G.S.; Wong, H.J. In vitro and ex vivo cellular antioxidant protection and cognitive enhancing effects of an extract of polygonum minus huds (lineminus ${ }^{\mathrm{TM}}$ ) demonstrated in a barnes maze animal model for memory and learning. BMC Complement. Altern. Med. 2014, 14, 161. [CrossRef] [PubMed]

21. Stohs, S.J.; Bagchi, D. Antioxidant, anti-inflammatory, and chemoprotective properties of acacia catechu heartwood extracts. Phytother. Res. 2015, 29, 818-824. [CrossRef] [PubMed]

22. Shilpa, K.; Krishnakumar, G.; Sooryaprakash, S. Phytochemical composition, antioxidant, and antibacterial activities of two Syzygium spp. J. Herbs Spices Med. Plants 2014, 20, 45-54. [CrossRef] 
23. Saha, M.R.; Dey, P.; Begum, S.; De, B.; Chaudhuri, T.K.; De Sarker, D.; Das, A.P.; Sen, A. Effect of acacia catechu (lf) willd. On oxidative stress with possible implications in alleviating selected cognitive disorders. PLoS ONE 2016, 11, e0150574. [CrossRef] [PubMed]

24. Thio, T.H.G.; Soroori, S.; Ibrahim, F.; Al-Faqheri, W.; Soin, N.; Kulinsky, L.; Madou, M. Theoretical development and critical analysis of burst frequency equations for passive valves on centrifugal microfluidic platforms. Med. Biol. Eng. Comput. 2013, 51, 525-535. [CrossRef] [PubMed]

25. Kazemzadeh, A.; Ganesan, P.; Ibrahim, F.; He, S.; Madou, M.J. The effect of contact angles and capillary dimensions on the burst frequency of super hydrophilic and hydrophilic centrifugal microfluidic platforms, a CFD study. PLoS ONE 2013, 8, e73002. [CrossRef] [PubMed]

26. Brand-Williams, W.; Cuvelier, M.-E.; Berset, C. Use of a free radical method to evaluate antioxidant activity. LWT-Food Sci. Technol. 1995, 28, 25-30. [CrossRef]

27. Tang, M.; Wang, G.; Kong, S.-K.; Ho, H.-P. A review of biomedical centrifugal microfluidic platforms. Micromachines 2016, 7, 26. [CrossRef]

28. Demello, A.J. Control and detection of chemical reactions in microfluidic systems. Nature 2006, 442, $394-402$. [CrossRef] [PubMed]

29. Mark, D.; Haeberle, S.; Roth, G.; Von Stetten, F.; Zengerle, R. Microfluidic lab-on-a-chip platforms: Requirements, characteristics and applications. In Microfluidics Based Microsystems; Springer: Berlin, Germany, 2010; pp. 305-376.

30. Altman, D.G.; Bland, J.M. Standard deviations and standard errors. BMJ 2005, 331, 903. [CrossRef] [PubMed]

31. Phonchai, A.; Kim, Y.; Chantiwas, R.; Cho, Y.-K. Lab-on-a-disc for simultaneous determination of total phenolic content and antioxidant activity of beverage samples. Lab Chip 2016, 16, 3268-3275. [CrossRef] [PubMed]

32. Papariello, G.; Janish, M. Diphenylpicrylhydrazyl as an organic analytical reagent in the spectrophotometric analysis of phenols. Anal. Chem. 1966, 38, 211-214. [CrossRef]

33. Molyneux, P. The use of the stable free radical diphenylpicrylhydrazyl (DPPH) for estimating antioxidant activity. Songklanakarin J. Sci. Technol. 2004, 26, 211-219.

34. Schaich, K.; Tian, X.; Xie, J. Hurdles and pitfalls in measuring antioxidant efficacy: A critical evaluation of ABTS, DPPH, and ORAC assays. J. Funct. Foods 2015, 14, 111-125. [CrossRef]

35. Lopez-Alarcon, C.; Denicola, A. Evaluating the antioxidant capacity of natural products: A review on chemical and cellular-based assays. Anal. Chim. Acta 2013, 763, 1-10. [CrossRef] [PubMed]

36. Uddin, S.M.; Ibrahim, F.; Sayad, A.A.; Thiha, A.; Pei, K.X.; Mohktar, M.S.; Hashim, U.; Cho, J.; Thong, K.L. A portable automatic endpoint detection system for amplicons of loop mediated isothermal amplification on microfluidic compact disk platform. Sensors 2015, 15, 5376-5389. [CrossRef] [PubMed]

37. Thiha, A.; Ibrahim, F. A colorimetric enzyme-linked immunosorbent assay (elisa) detection platform for a point-of-care dengue detection system on a lab-on-compact-disc. Sensors 2015, 15, 11431-11441. [CrossRef] [PubMed]

(C) 2018 by the authors. Licensee MDPI, Basel, Switzerland. This article is an open access article distributed under the terms and conditions of the Creative Commons Attribution (CC BY) license (http:// creativecommons.org/licenses/by/4.0/). 